\title{
¿HAY UNA TEORÍA NO IDEALISTA DE LA CONSTITUCIÓN?
}

\author{
Mariano Crespo \\ Facultad de Filosofía \\ Pontificia Universidad Católica de Chile \\ mcrespos@uc.cl
}

\begin{abstract}
Resumen
Este artículo intenta mostrar que una teoría de la constitución de los objetos del conocimiento no conlleva necesariamente una teoría idealista del conocimiento. El punto de partida es, por un lado, la reconstrucción de los principales elementos de la teoría husserliana de la constitución y, por otro, el análisis de un argumento usado por Husserl en las Investigaciones lógicas a fin de probar la existencia de objetos ideales. En este orden de cosas, Antonio Millán-Puelles ha mostrado la posibilidad de una "génesis intencional" de los objetos en el sentido del surgimiento de éstos ante la conciencia.
\end{abstract}

Palabras Clave: constitución, objetos ideales, entes de razón, realismo, génesis intencional, génesis real, fenomenología.

\section{Abstract}

This paper tries to show that there is at least a sense in which one can defend the idea that the constitution of objectivity is compatible with metaphysical realism. The point of departure is, on the one hand, the reconstruction of the main elements of Husserl's theory of constitution and, on the other hand, the analysis of an argument used by this philosopher in Logical Investigations to prove the existence of ideal objects. As Antonio Millán-Puelles has showed, one can talk of an "intentional genesis" of objects in the sense of their arising before consciousness.

KEYWORDS: Constitution, beings of reason, ideal objects, realism, intentional genesis, real genesis, phenomenology.

Una de las cuestiones que han ocupado a la filosofía de todos los tiempos es
el modo de entender esa peculiar relación en que consiste el conocimiento.
¿En qué consiste propiamente conocer? ¿Qué estatuto ontológico poseen aquel que
conoce y lo conocido por éste? ¿Qué tipo de relación existe entre ambos? ¿Qué sucede
cuando tiene lugar un acto cognoscitivo? ¿Qué lugar ocupa la teoría del conocimiento
dentro de la filosofía? ¿Depende esta disciplina de otras disciplinas filosóficas o extra
filosóficas o es, por el contrario, independiente de ellas? ¿Hasta qué punto el sujeto 
que conoce moldea de alguna forma lo conocido o simplemente lo deja aparecer ante él? ¿Se trata de una relación en la que el sujeto desempeña un papel activo o simplemente es testigo pasivo de lo que ante él se da? ${ }^{1}$

Estas y otras preguntas similares relacionadas con la clarificación de conceptos epistemológicos fundamentales estuvieron en el centro de la labor filosófica de la fenomenología iniciada por Edmund Husserl en los albores del siglo XX, con la publicación de la primera edición de Investigaciones lógicas (1900-1901). En este orden de cosas, Robert Sokolowski ha destacado que uno de los temas fundamentales de esta obra es justamente el esclarecimiento de la relación entre la subjetividad del conocer y la objetividad del objeto del conocimiento ${ }^{2}$. A este tema pertenecen, por ejemplo, los diversos análisis de la intencionalidad, como, por ejemplo, la diferencia entre nombrar, juzgar y sus correlatos intencionales, los análisis de los actos en los cuales se constituyen las significaciones, las explicaciones sobre el carácter fundado de la intuición categorial, etc. Estas diversas explicaciones del modo en el que la objetividad del contenido del conocimiento es comprendida por la subjetividad se agrupan bajo el título común de "constitución de la objetividad por la subjetividad"3. Este problema y los diferentes modos de interpretarlo representan la "crux" de la fenomenología husserliana.

Relacionado con lo anterior, algunos autores de raigambre realista han considerado que el descubrimiento de la problemática de la constitución ponía a Husserl irremediablemente en el sendero de un idealismo trascendental. Hablar de "constitución" de los objetos por parte de la conciencia incluiría determinados matices "creativos", los cuales entrarían en conflicto con el objetivismo de la primera edición de Investigaciones lógicas. Sin embargo, nada parece estar más lejos de las intenciones del fundador del método fenomenológico. De hecho, en un conocido texto de una carta del 25 de enero de 1903 a W. E. Hocking, Husserl escribe: "La expresión 'los objetos se constituyen' en un acto significa siempre la propiedad del acto de representarse el objeto, ¡no 'constituirlo' en sentido propio!”4. Por su parte, Walter Biemel, en su conocido artículo "Las fases decisivas en el desarrollo de la filosofía de Husserl", apunta, a mi juicio muy acertadamente, a la poca frecuencia

Para el tratamiento de algunas de estas cuestiones, véase Ingarden 2006.

Sokolowski 1970, p. 39 ss.

También Sokolowski 2000, pp. 92-93 defiende esta interpretación "neutral" de la constitución: "In phenomenological terminology, the establishment of categorial objects is called their constitution. The term constitution should not be taken to mean anything like a creation or an imposition of subjective forms on reality. In phenomenology to constitute a categorical object means to bring it to light, to actualise its truth. We cannot make an object man anything we wish. We can bring a thing to light only if the thing offers itself in a certain light".

4 "Der... Ausdruck, daß sich in einem Akte 'Gegenstände konstituieren', besagt immer die Eigenschaft des Aktes, den Gegenstand vorstellig zu machen: nicht 'konstituieren' im eigentlichen Sinn!”. Citado por U. Panzer en Husserl 1984, p. XVIII, nota 2. 
con la que se ha intentado captar la coherencia interna del pensamiento del fundador de la fenomenología. Según Biemel se impone una consideración genética de la fenomenología que busque no solamente las significaciones establecidas, sino también el proceso de su establecimiento. El hilo conductor de este proceso es la idea de que para iluminar la esencia de una cosa es necesario remontarse al origen de su significación en la conciencia y a la descripción de este origen (cf. Biemel 1968, p. 72). Esta idea recorrería la obra filosófica de Husserl desde la Filosofía de la Aritmética hasta sus últimas obras. Incluso Edith Stein, una de las personas que sintieron que la aparición de Ideas I suponía un cambio con respecto al rumbo objetivista marcado por las Investigaciones lógicas, en una recensión de las Meditaciones Cartesianas escribe lo siguiente:

“Existe alguna posibilidad desde la philosophia perennis de apropiarse de la problemática de la constitución fenomenológica sin adoptar por ello lo que se ha denominado idealismo trascendental de la fenomenología?" (Stein 1962, p. 35).

Esta pregunta de Edith Stein está estrechamente vinculada con la cuestión que constituye el título de este artículo, a saber, ¿existe una teoría no idealista de la constitución de los objetos? O dicho de otro modo ¿está toda teoría de la constitución de los objetos de la conciencia esencialmente vinculada a una teoría idealista del conocimiento en el sentido de una construcción de los mismos?

No es fácil responder con brevedad a esta cuestión. Un tratamiento detenido y sistemático de los aspectos con ella relacionados superarían los límites de esta contribución. Aquí tan solo me quiero referir a algunos puntos esenciales e indicar algunas líneas de investigación que merecerían ser desarrolladas en el futuro. Para ello procederé del siguiente modo. En primer lugar, y después de unas palabras introductorias, haré un breve resumen de la teoría husserliana de la constitución de los objetos lógicos. En segundo lugar, me ocuparé críticamente de un argumento para probar la existencia de los objetos lógicos que Husserl ofrece en las Investigaciones lógicas. Por último, ofreceré un esbozo de los fundamentos de la teoría de la "génesis intencional" de los objetos puros de A. Millán-Puelles (1990), como posible respuesta a la cuestión planteada por Edith Stein.

Este interés en la relación entre subjetividad del conocimiento y objetividad del contenido del mismo al que apunta la problemática de la constitución, lo encontramos también en el juicio que Husserl emite sobre la situación de la lógica formal en su época. Según este pensador, ésta requiere una reflexión radical. ¿Se necesita simplemente una purificación del estado de la misma o existe un fin positivo de esta reflexión radical? En el ámbito de la lógica formal, se mostraría especialmente la urgencia de una explicación intencional del sentido propio de la misma (Husserl 1962, Einleitung). Ésta "parte de las formaciones teóricas que nos ofrece la experiencia 
histórica considerada en su conjunto, esto es, de su contenido objetivo tradicional; y las retrae a la intención viva de los lógicos de la que surgieron como formaciones significativas. Lo que es inseparable de lo anterior: se retrae a la intencionalidad de los cientificos, de la cual surgieron los componentes objetivos de las teorías científicas concretas: pues el lógico se orienta por las ciencias existentes. Preguntamos: ¿a qué tiende propiamente la intencionalidad que revive en cada nueva comprensión efectiva? La exposición reflexiva, en cuanto clarificación crítica, ha de dar respuesta” (Ibíd.).

La lógica tradicional no habría planteado sus cuestiones fundamentales de un modo suficientemente radical. Habría descuidado, sobre todo, un elemento de la mencionada relación entre la subjetividad del conocimiento y la objetividad del contenido de éste. El tema de la dirección objetiva de la lógica son los resultados producidos por la actividad de la razón (Husserl 1974, p. 37). Se trata de "resultados permanentes" (bleibende Erwerbe) que sobrepasan la esfera de la presencia a la conciencia. Ejemplo de estos productos son los conceptos, los juicios, los razonamientos, las teorías, etc. El "error" o la falta de radicalidad de la lógica tradicional consistiría, según Husserl, en haber estado "encadenada" a las Gestalten teóricas objetivas y en no haber investigado la subjetividad intencional en la cual estos resultados se constituyen como unidades sintéticas.

En Lógica formal y trascendental encontramos precisamente un interés creciente por la subjetividad del conocer. En esta obra comienza el camino de una fenomenología estática a una fenomenología genética y se esboza el programa de un análisis genético de los objetos lógicos. Ya sabemos que este camino conduce a la pregunta acerca del origen de dichos objetos. En este orden de cosas, en la segunda sección de esta obra, Husserl emprende la tarea de una fundamentación trascendental de la lógica que según él no ha de ser confundida con un psicologismo. Esta tarea es desarrollada posteriormente en Experiencia y Juicio con motivo del análisis del juicio predicativo. En este orden de cosas, Husserl señala que la pregunta por el carácter de la evidencia de los objetos es la pregunta por la datitud evidente de los individuos. La experiencia se define pues primariamente como una relación directa a lo individual ${ }^{5}$. El presupuesto de estos juicios es la datitud evidente de los objetos individuales. Esta evidencia es la evidencia pre-predicativa. Para comprender mejor esta evidencia, es necesario ser consciente de que los objetos construyen un cierto trasfondo del campo de nuestra conciencia. Podemos pues decir que antes de todo acto de conocimiento están ahí como objeto del conocimiento o de la experiencia. Este "entorno" o "mundo" caracteriza un ámbito de pre-datitud. Toda actividad cognoscitiva presupone este ámbito de pre-datitud. Podemos incluso decir que toda actividad cognoscitiva presupone como suelo universal un mundo en cuya existencia creemos ${ }^{6}$.

5 "Daher sind die an sich ersten Urteile als Urteile mit individuellen Substraten, Urteile über Individuelles, die Erfahrungsurteile" (Husserl 1948, p. 21).

$6 \quad$ "So affiziert alles Seiende, das uns affiziert, auf dem Boden der Welt, es gibt sich uns als vermeintlich Seiendes; und Erkenntnistätigkeit, Urteilstätigkeit, geht darauf, es zu prüfen, 
El siguiente paso consiste en retroceder al mundo pre-dado de la experiencia, al mundo en el cual ya vivimos (Lebenswelt). Este mundo es el "suelo" de todo rendimiento cognoscitivo y nos es pre-dado como "preñado" con los resultados de los rendimientos lógicos. Este retroceso al mundo de la vida requiere también una purificación de las diversas "idealizaciones" que están conectadas con él. Esta "purificación" no es una tarea que la psicología pueda asumir, puesto que ésta se ocupa de vivencias de sujetos de nuestro mundo. Por ello, el develamiento de las diversas idealizaciones y resultados de los rendimientos subjetivos significa un retroceso a lo subjetivo, a una subjetividad que no puede ser objeto de estudio por parte de la psicología. Se trata de una subjetividad trascendental.

Después de haber esbozado los fundamentos de la interpretación husserliana de la constitución de los objetos lógicos, quisiera volver a la primera edición de las Investigaciones lógicas. Con ayuda del filósofo español Antonio Millán-Puelles quisiera reconstruir y considerar críticamente un argumento de Husserl para probar la existencia de objetos ideales. En la primera Investigación lógica escribe Husserl:

“(...), suelen llamarse 'sin sentido' o suele negársele significación (en giros equivalentes) a expresiones contradictorias o gravadas con evidentes incompatibilidades, como cuadrado redondo. (...) Marty objeta (...): 'si las palabras no tuviesen sentido, ¿cómo íbamos a poder comprender la pregunta de si existe tal o cual y negarla? Incluso para rechazarla necesitamos representar de uno u otro modo esa materia contradictoria'. (...) 'Si a esos absurdos se les llama 'sin sentido', esto no puede significar sino que no tienen evidentemente ningún sentido racional'. Estas objeciones son totalmente certeras, en cuanto que la forma de exposición en los citados investigadores permite suponer que la falta de sentido auténtica (...) ha sido por ellos confundida con la imposibilidad a priori de un sentido impletivo (apriorischen Unmöglichkeit eines erfüllenden Sinnes). Una expresión tiene, pues, en este sentido una significación cuando a su intención corresponde un sentido posible; $\mathrm{o}$, dicho con otras palabras, la posibilidad de una intuición unitaria" (1984a, A 55-56).

Así vemos que "expresiones absurdas" como cuadrado redondo tienen para Husserl un sentido, un significado. El ser de un cuadrado redondo es su ser pensado por nosotros. Husserl explica el carácter ontológico de lo ficticio y de lo absurdo:

ob es als das wie es sich gibt, wie es vorweg vermeint ist, wahrhaft ist und wahrhaft so und so seiendes ist. Welt als seiende Welt ist die universale passive Vorgegebenheit aller urteilenden Tätigkeit, alles einsetzenden theoretischen Interesses" (Husserl 1948, p. 26). 
"Naturalmente, no es nuestro propósito poner en un mismo plano el ser de lo ideal y el ser pensado de lo ficticio o contra sentido. Este último no existe. De él no puede decirse nada categóricamente en sentido propio. Y si a pesar de ello hablamos de él como si fuera, como si tuviera su modo propio de ser, el modo 'meramente intencional', resulta esta manera de hablar -considerada más exactamente- una manera de hablar impropia" (1984a, A 124).

Utilizando una distinción llevada a cabo por Millán-Puelles en su Teoría del Objeto Puro (1990, p. 211), podemos resumir la posición de Husserl afirmando que el absurdo es un verdadero objeto del pensar, pero no un objeto que verdaderamente es, en el sentido de un objeto cuyo ser no se limita a nuestro ser pensado. Acudiendo de nuevo a Husserl, nos encontramos con su argumento principal para mostrar la existencia de los objetos ideales:

"En cambio, los objetos ideales existen verdaderamente. Es evidente que no solo tiene sentido hablar de tales objetos (por ejemplo: del número 2, de la cualidad rojez, del principio de contradicción, y otros semejantes) y representarlos como dotados de predicados, sino que también aprehendemos intelectivamente ciertas verdades categóricas, que se refieren a estos objetos ideales. Si estas verdades valen, tiene que existir todo aquello que presupone objetivamente su validez (Gelten diese Wahrheiten, so muß all das sein, was ihre Geltung objektiv voraussetzt). Si veo con intelección que 4 es número par, que el predicado enunciado conviene realmente al objeto ideal 4 , entonces este cobjeto no puede ser una mera ficción, una mera ,façon de parler', una nada" (1984a, A 124/B 125).

Parece, pues, que el fundamento de la existencia de los objetos ideales reside, según el fundador de la fenomenología, en su capacidad de ser sujetos de juicios categóricos. Millán-Puelles objeta que Husserl, en su esfuerzo por distinguir entre objetos absurdos y objetos ideales, habría ido muy lejos. Husserl, según el filósofo español, no habría visto que los objetos ideales son verdaderos objetos del pensar, pero no objetos que verdaderamente son. Este tipo de objetos son precisamente los "objetos puros". Millán-Puelles pone de manifiesto cómo es posible llevar a cabo juicios verdaderos sobre objetos puros, esto es, sobre objetos que no existen verdaderamente, que no tienen una existentia in re. De este modo, llega a la siguiente conclusión:

"El número 2, como cualquier otro número abstracto o universal, tiene innegablemente un fundamento in re, pero no existe, ni puede existir, en su estado de universalidad o de abstracción. Lo existente es determinado, concreto, individual. Por la misma razón no es la rojez tampoco un verdadero ente. Hay cosas rojas y, en ellas, las respectivas rojeces individuales, pero la 'rojez' así en abstracto, 'desindividualizada', es un producto mental, un ens rationis, si bien tiene, sin duda, en las rojeces concretas un fundamento in re. Y, por su parte, el principio de contradicción no deja de ser-verdadero por carecer de un verdadero ser. Como cualquier enuntiabile, es una construcción mental, una estructura carente de entidad efectiva, aunque no un engendro del capricho, dado que cuenta con un fundamento in re. Y justamente por hallarse, en cambio, desprovistos de este tipo de fundamento es por lo que los objetos imposibles o absurdos han de 
inscribirse en un plano específicamente diferente del que es propio de los objetos ideales según los concibe Husserl" (Millán-Puelles 1990, p. 212).

Los objetos "verdaderamente existentes", los objetos reales, existen in actu, tienen un ser que va "más allá" de su ser pensados por una conciencia. Tienen, pues, un ser "transobjetual", un sistere extra cogitationem. Por el contrario, los objetos irreales o puros no existen extra cogitationem. No son res obiecta, sino mero obiectum ${ }^{7}$. Si decimos que "hay" objetos irreales, este "hay" significa un mero ser objetual. «Cogito, ergo sum, sí; pero no cogitatum, ergo est» (Millán-Puelles 1990, p. 227).

Ciertamente, los por Husserl denominados "objetos ideales" no tienen el estatuto ontológico de los objetos absurdos o ficticios. Mientras que los últimos no tienen un fundamentum in re, los entia rationis -en el lenguaje de la filosofía escolástica- poseen un fundamento tal. La cuestión que aquí planteamos no es tanto si esta teoría, aquí tan solo esbozada, de Millán-Puelles que tiene su origen en la filosofía de realistas clásicos como Aristóteles, Tomás de Aquino, Francisco Suárez, etc., presenta ciertas semejanzas con la teoría husserliana de la constitución, sino si en el seno de una filosofía realista puede hablarse de constitución en el sentido de un "crear" meramente intencional, que no es un crear real de algo, sino una "construcción" de las objetividades para la conciencia.

El ser de los objetos reales, transobjetuales, no se reduce, pues, a los actos en virtud de los cuales estos objetos son, valga la redundancia, "objetivados" para una conciencia. Ésta es, como es sabido, una afirmación fundamental de todo realismo metafísico. Pero no solamente eso. El realismo metafísico no se reduce al reconocimiento de esta irreductibilidad. La irreductibilidad de lo irreal a todo acto que lo representa desempeña también un papel importante. El reconocimiento de lo irreal implica, como veíamos, la distinción entre lo "verdaderamente existente" y el "mero darse objetualmente". A esta diferencia corresponde la distinción entre la génesis física real de los actos de la representación y la mera génesis intencional de los objetos irreales (Millán-Puelles 1990, p. 613 ss.). Esta génesis meramente intencional no consiste en un crear real de algo, sino, como decíamos, en la aparición de determinadas

7 “(...) lo irreal no tiene otra vigencia que su mera objetualidad, vale decir, su puro y simple darse como objeto ante una subjetividad consciente en acto. En ningún sentido es res obiecta, sino tan solo obiectum: mero ser-ante-la-conciencia y para ella. Fuera de esto no es nada (si es que en verdad el ser eso, y absolutamente nada más, merece llamarse ser). De ahí su constitutiva oposición a lo real qua real, que es lo transobjetual en el sentido de que su ser no se agota, ni consiste tampoco bajo ningún aspecto, en estar-siendo-objeto ante una subjetividad consciente en acto" (Millán-Puelles 1990, p. 21). 
objetividades ante la conciencia. Se trata, pues, de una "actividad" del sujeto que no es en modo alguno arbitraria o haya de ser entendida en sentido psicologista ${ }^{8}$.

La presencia de los objetos irreales no es menos "verdadera" que la presencia de los objetos reales. Pero los objetos irreales son solo objetos de una mera génesis intencional. Esta "génesis" no es un "producir", sino que se trata del dejar aparecer ante la conciencia. Lo aquí enigmático es que ciertos objetos irreales aparecen ante nuestra conciencia mediante la producción real de ciertos actos ${ }^{9}$. La utilización dentro del realismo metafísico clásico de términos como fieri, consurgere, fabricatio, entre otros para caracterizar la "constitución" de los objetos irreales ante la conciencia, no ha de entenderse, según Millán-Puelles, en modo psicologista alguno. Los términos latinos que acabamos de mencionar fueron empleados precisamente por Francisco Suárez para denominar los actos en virtud de los cuales se dan los entia rationis.

Así escribe Suárez:

"Intellectus est causa efficiens entium rationis; efficit autem illa efficiendo solum aliquam cogitationem vel conceptum suum, ratione cuius dicitur ens rationis habere esse obiective in intellectu" (Suárez 1866, Disp. LIV, sect. 2, n.4).

8 En un esclarecedor texto, Edith Stein se refiere a esta actividad de generación intencional, no real, de la conciencia: "Wenn wir von einer Auffassungstätigkeit des Subjekts sprechen, so ist das nicht so zu deuten, als ob das Subjekt nach freier Willkür ein form- und gestaltloses Material 'bearbeitete': ein Demiurg, der aus der Materie die Welt schafft, der das Chaos zum Kosmos gestaltet. In der Auffassung der realen Welt ist das Subjekt nicht frei. Es ist einmal eine bestimmte Struktur des sinnlichen Materials erforderlich, damit sich auf seinem Grunde ein intentionales Erlebnis, eine Gegenstandsauffassung aufbauen könne. Andererseits stellt sich -wenn ein so und so gearteter Verlauf sinnlicher Daten auftritt- die Gegenstandsauffassung ein, ohne dass das Subjekt die Freiheit hätte, sie zu unterlassen. Eine feste Gesetzlichkeit beherrscht die Konstitution der Gegenständlichkeiten, d.h., ihren Aufbau für das Bewußtsein" (Stein 1991, pp. 85-86).

$9 \quad$ "La génesis intencional es génesis del objeto, por más que lo constituido en virtud de ella no esté siendo presente, en calidad de objeto a su vez. Esta génesis es meramente intencional porque no estriba en producir efectiva o realmente algo, pero es, no obstante, verdadera génesis porque hace que algo surja ante nosotros, no siendo a su vez ese algo el acto mismo de la representación, el cual emerge físicamente en la conciencia (o, mejor, en la subjetividad que la ejerce), de tal modo que su surgir mismo es una génesis óntica, real, pero no intencional. La génesis meramente intencional del acto mismo de la representación acontece con la génesis físicamente real de la reflexión sobre él, en la cual es también donde acontece la explícita representación de la objetualidad ut sic" (Millán-Puelles, 1990, pág. 618). “Toda génesis intencional es una efectiva suscitación de algo 'ante’ y 'para' una subjetividad activamente consciente (no solo apta para la conciencia), y es por ello una génesis auténtica, aunque no produce nada real. Ahora bien, precisamente porque no produce nada real, la génesis intencional es verdadera génesis solo por su solidaridad con el efectivo producirse de la realidad de un acto de representación. Si este acto no fuera verdaderamente 'producido' o 'generado' en la más fuerte acepción de los dos términos, nada irreal quedaría verdaderamente suscitado” (Millán-Puelles, 1990, p. 625). 
Aquí nos encontramos con una clara distinción entre el pensamiento (cogitatio), que el intelecto produce y el ens rationis, del cual se afirma que tiene el ser objetivo (esse obiectivum) in intellectu. Esto significa que el ens rationis en el intellectus no tiene un esse auténtico. Si el ens rationis tiene el ser objetivo solo in intellectu, esto significa que tiene el esse obiectivum merced a un acto del intelecto. Se trata pues de una efficentia de este acto, de una génesis, pero lato modo.

Ciertamente, el idealismo trascendental de Husserl no es compatible con el realismo metafísico que está tras estas teorías de Suárez y Millán-Puelles. Además, habría que investigar si el sentido de "irreal" en la terminología husserliana y en la teoría del objeto puro es el mismo. Existe sobre todo una diferencia fundamental entre la teoría de la constitución de Husserl y la teoría de la génesis intencional de los objetos puros. La teoría del objeto puro afirma (a) que el ser corresponde al mundo considerado éste en sí mismo y que éste es relativo a la conciencia solo en cuanto objeto. Como Millán-Puelles muestra, esta diferencia no puede ser hecha en Husserl. ¿Por qué? Porque la epoché nos prescribe poner en suspenso toda afirmación o negación del valor existencial de los objetos del mundo (cf., por ejemplo, Hua III/1, p. 56).

En este trabajo hemos intentado ofrecer un esbozo de respuesta positiva a la pregunta de Edith Stein mencionada al inicio del mismo. Parece, pues, que no toda teoría de la constitución de los objetos de conocimiento conlleva necesariamente una teoría idealista del conocer. Es más, uno de los requisitos del realismo metafísico es, justamente, una teoría tal. La teoría del objeto puro de Antonio Millán-Puelles, con su distinción entre génesis intencional y génesis real, es un ejemplo de ello.

\section{Referencias bibliográficas}

Biemel, W. (1968), "Las fases decisivas en el desarrollo de la filosofía de Husserl", Husserl. Tercer Coloquio filosófico de Royaumont (G. Maci, Übers., S. 35-57). Buenos Aires: Paidós.

Husserl, E. (1948), Erfahrung und Urteil. Hamburg: Claassen.

(1974), Formale und transzendentale Logik. Versuch einer Kritik der logischen Vernunft (Bd. Husserliana XVII). (P. Janssen, Hrsg.). The Hague: Martinus Nijhoff.

(1950), Ideen zu einer reinen Phänomenologie und phänomenologische Philosophie (Bd. Husserliana III/1). (W. Biemel, Hrsg.). The Hague: Maartinus Nijhoff. 
(1962), Lógica formal y trascendental (L. Villoro, Übers.). México D. F.: Universidad Nacional Autónoma de México.

(1975), Logische Untersuchungen. Erster Teil. Prolegomena zur reinen Logik (E. Holenstein, Hrsg.). The Hague: Martinus Nijhoff.

(1984a), Logische Untersuchungen. Zweiter Band. Erster Teil (Bd. Husserliana XIX/1). (U. Panzer, Hrsg.). The Hague: Martinus Nijhoff.

(1984b), Logische Untersuchungen. Zweiter Teil. Zweiter Band (Bd. Husserliana XIX/2). (U. Panzer, Hrsg.). The Hague: Martinus Nijhoff.

Ingarden, R. (2006), Sobre el peligro de una petitio principii en la teoría del conocimiento (M. Crespo, Übers.). Madrid: Ediciones Encuentro.

Millán-Puelles, A. (1990), Teoría del objeto puro. Madrid: Rialp.

Sokolowski, R. (2000), Introduction to Phenomenology. Cambridge: Cambridge University Press.

(1970), The Formation of Husserl's Concept of Constitution. The Hague: Martinus Nijhoff.

Stein, E. (1962), "Husserls transzendentale Phänomenologie”, en E. Stein, L. Gelber, \& R. Leuven (Hrsg.), Edith Steins Werke (Bd. VI). Louvain: Nauwelaerts.

(1991), Einführung in die Philosophie (Edith Steins Werke Ausg., Bd. XII). (L. Gelber, \& M. Linssen, Hrsg.). Freiburg: Herder.

Suárez, F. (1866), "Disputationes Metaphysicae”, en F. Suárez, \& C. Berton (Ed.), Opera Omnia (Vol. XXVI). París: Bibliopolam Editorem. 